\title{
ESTUDIO CINÉTICO DE LA DESCOMPOSICIÓN CATALIZADA DE PERÓXIDO DE HIDRÓGENO SOBRE CARBÓN ACTIVADO
}

\author{
Elihu Paternina, Juan M. Arias y Daniel Barragán* \\ Departamento de Química, Facultad de Ciencias, Universidad Nacional de Colombia, Carrera 30 No 45-03, Bogotá, Colombia
}

Recebido em 23/5/08; aceito em 7/11/08; publicado na web em 26/2/06

\begin{abstract}
KINETIC STUDY OF THE CATALYZED DECOMPOSITION OF HYDROGEN PEROXIDE ON ACTIVATED CARBON. The kinetic study of decomposition of hydrogen peroxide catalyzed by activated carbon was carried out. The effect of concentrations of reactants and temperature were experimentally studied. Kinetic data were evaluated using differential method of initial rates of reaction. When a typical kinetic law for reactions in homogeneous phase is used, first order of reaction is obtained for hydrogen peroxide and activated carbon, and activation energy of $27 \mathrm{~kJ} \mathrm{~mol}^{-1}$ for the reaction was estimated. Experimentally was observed that surface of activated carbon is chemically modified during decomposition of hydrogen peroxide, based on this result a scheme of reaction was proposed and evaluated. Experimental data fits very well to a Langmuir- Hinshelwood kinetic model and activation energy of $40 \mathrm{~kJ} \mathrm{~mol}^{-1}$ was estimated for reaction in heterogeneous phase.
\end{abstract}

Keywords: decomposition of hydrogen peroxide; initial rate of reaction; kinetic law of Langmuir-Hinshelwood.

\section{INTRODUCCIÓN}

La descomposición catalizada del peróxido de hidrógeno (DCPH), tanto en fase homogénea como heterogénea, es una reacción ampliamente estudiada debido a la alta reactividad de la molécula y al poder oxidante de los productos intermediarios de reacción. ${ }^{1,2}$ La DCPH en la superficie de sólidos ocurre selectivamente en sitios activos básicos o reductores o en sitios donde estén involucrados iones de metales tales como, hierro, cerio, manganeso, cobre, cobalto, vanadio, etc., los cuales pueden estar en diferentes estados de oxidación y/o formando complejos. ${ }^{3-6}$ La selectividad para la DCPH en la superficie de un sólido es de utilidad para evaluar y comparar la actividad catalítica de sólidos modificados. ${ }^{3,4}$ Esta evaluación se realiza asumiendo que la cinética de la DCPH obedece a una ley de pseudo-primer orden, Ecuación 1, a partir de la cual se calcula y se compara la constante de velocidad observada $\left(k_{\text {obs }}\right)$

$v_{\left[\mathrm{H}_{2} \mathrm{O}_{2}\right]_{0}}=-\frac{d\left[\mathrm{H}_{2} \mathrm{O}_{2}\right]_{0}}{d t}=k_{\text {obs }}\left[\mathrm{H}_{2} \mathrm{O}_{2}\right]_{0}$

donde $\left[\mathrm{H}_{2} \mathrm{O}_{2}\right]_{0}$ es para la concentración inicial de peróxido de hidrógeno.

Lin y Gurol en 1998 estudiaron la DCPH catalizada por oxido de hierro, ${ }^{7}$ y Huang et al. en 2003 estudiaron la descomposición catalizada por carbones activados modificados, ${ }^{8}$ encontrando que la tendencia de los datos experimentales se ajustan a una ley cinética de tipo Langmuir-Hinshelwood, como la que se muestra en la Ecuación 2,

$v_{\left[\mathrm{H}_{2} \mathrm{O}_{2}\right]_{0}}=-\frac{d\left[\mathrm{H}_{2} \mathrm{O}_{2}\right]_{0}}{d t}=\frac{k_{v e l} \mathrm{CAT}\left[\mathrm{H}_{2} \mathrm{O}_{2}\right]_{0}}{1+k_{h}\left[\mathrm{H}_{2} \mathrm{O}_{2}\right]_{0}}$

donde $k_{v e l}$ y $k_{h}$ se interpretan como la constante cinética de reacción y la de formación de reactivos a partir de intermediarios, respectivamente; $C A T$ es la relación masa/volumen de carbón activado y $\left[\mathrm{H}_{2} \mathrm{O}_{2}\right]_{0}$ es la concentración inicial de peróxido de hidrógeno. Sin embargo estos

*e-mail: dabarraganra@bt.unal.edu.co estudios no se realizaron de manera sistemática, esto es, se llevaron a cabo a una sola temperatura y una sola relación masa-volumen de catalizador y además se realizaron pocos experimentos en un pequeño intervalo de concentraciones iniciales de peróxido de hidrógeno $\left(1,8 \times 10^{-3}\right.$ a $\left.4,0 \times 10^{-2} \mathrm{~mol} \mathrm{~L}^{-1}\right)$.

En los trabajos publicados hasta el momento a cerca del estudio cinético de la descomposición del peróxido de hidrógeno, no se ha encontrado un estudio sistemático del efecto de las concentraciones iniciales de reactivos en un intervalo amplio, ni del efecto de la temperatura; lo cual se constituye en el objetivo principal de este trabajo.

\section{PARTE EXPERIMENTAL}

\section{Reactivos}

Peróxido de hidrógeno Panreac 30\%. Carbón activado J.T. Baker (CAS No. 7440-44-0) de origen vegetal y lavado ácido, con área superficial de microporo determinada por el método de DubinninRaduskevich de 1154 m²/g. Al carbón se le realizo la siguiente caracterización: número de yodo de 1035, índice de azul de metileno de 21,35 y caracterización de los grupos funcionales por el método de Bohem, con el siguiente resultado: grupos carboxílicos $1,22 \times 10^{-1} \mathrm{meq}$ $\mathrm{g}^{-1}$, lactonas $2,44 \times 10^{-1} \mathrm{meq} \mathrm{g}^{-1}$, fenoles $1,51 \times 10^{-1} \mathrm{meq} \mathrm{g}^{-1}$, carbonilos $1,24 \times 10^{-2}$ meq $\mathrm{g}^{-1} \mathrm{y}$ grupos básicos totales $1,12 \mathrm{meq} \mathrm{g}^{-1}$.

\section{Metodología}

Las reacciones de DCPH se llevaron a cabo en una celda de vidrio con doble pared para control de temperatura. La reacción se siguió mediante medida barométrica con un sensor diferencial de presión (Motorola MPX 2010 de 10,0 kPa). Para calcular la cantidad de oxígeno molecular producido en función del tiempo los datos de presión se corrigieron para tener en cuenta la presión de vapor de agua, la solubilidad del oxígeno y la constante de Henry del gas en función de la temperatura.

La mezcla de reacción se mantuvo siempre en $50 \mathrm{~mL}$. La reacción de DPCH se inicia con la adición de la solución de peróxido de hidrógeno, a la misma temperatura a la que se encuentra la mezcla 
agua carbón activado.

Con el fin de obtener más información de la naturaleza química del carbón activado, en relación a la acidez-basicidad y naturaleza redox de la misma, se estudio el efecto del pH de la solución y la modificación química de la superficie del carbón en la DCPH. Para una composición fija de la mezcla de reacción con concentración de peróxido $0,100 \mathrm{~mol} \mathrm{~L}^{-1}$ y una $C A T$ igual a $2,00 \times 10^{-3} \mathrm{~g} \mathrm{~mL}^{-1}$ a una temperatura de $298 \mathrm{~K}$, se encontró que: en el intervalo, de 2-10 unidades de $\mathrm{pH}$ de la solución la velocidad inicial de reacción no varía de manera significativa. Este resultado es consistente con la composición de grupos funcionales en el carbón activado dada por la titulación de Bohem, donde los grupos ácidos reactivos se encuentran en muy baja proporción comparados con los grupos básicos. Oxidantes fuertes como el permanganato de potasio y el peróxido de hidrógeno, modifican sustancialmente la superficie del carbón afectando la DCPH (Figura 1), lo cual permite concluir la presencia de grupos reductores en la superficie del carbón como sitios activos para la DCPH. El tratamiento químico de la superficie del carbón activado se realizó de la siguiente manera: con $\mathrm{HNO}_{3}$ de concentración $5 \mathrm{~mol} \mathrm{~L}^{-1}$ durante $6 \mathrm{~h}$, con $\mathrm{KMnO}_{4}$ de concentración $0,08 \mathrm{~mol} \mathrm{~L}^{-1}$ en medio ácido de $\mathrm{H}_{2} \mathrm{SO}_{4}$ de concentración $0,5 \mathrm{~mol} \mathrm{~L}^{-1}$ durante $4 \mathrm{~h}$ y con $\mathrm{H}_{2} \mathrm{O}_{2}$ el $30 \%$ durante 72 h. Al final de cada tratamiento el carbón activado se colocó en canastillas de alfa celulosa en extractores Soxhlet durante una semana hasta garantizar $\mathrm{pH}$ constante. Finalmente el carbón fue secado a $110{ }^{\circ} \mathrm{C}$ hasta peso constante y luego almacenado en desecador.

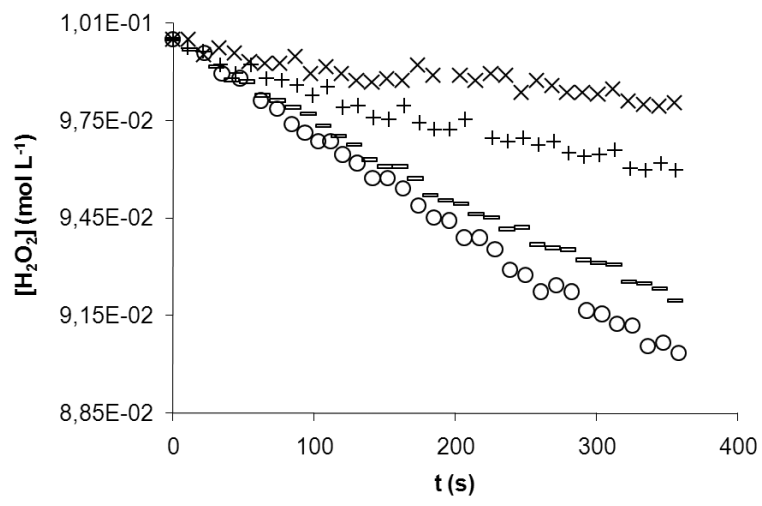

Figura 1. Descomposición de peróxido de hidrógeno sobre carbón activado (CA) químicamente modificado. (O) CA J. T. Baker sin modificar, (-) CA modificado con $\mathrm{HNO}_{3}$, (+) CA modificado con $\mathrm{H}_{2} \mathrm{O}_{2}$, (X) CA modificado con $\mathrm{KMnO}_{4}$. Condiciones iniciales: $\mathrm{CAT}=2,00 \times 10^{-3} \mathrm{~g} \mathrm{~mL}^{-1},\left[\mathrm{H}_{2} \mathrm{O}_{2}\right]_{0}=0,100 \mathrm{~mol}$ $L^{-1}$ y $T=298 K$

\section{Tratamiento cinético de los datos}

El estudio cinético se realizó por el método diferencial de velocidades iniciales. Para obtener la velocidad inicial, el conjunto de datos experimentales fue sometido a los siguientes tratamientos numéricos para garantizar la confiabilidad del valor obtenido: ajuste de diferentes intervalos de datos a polinomios de orden $3,4,5$ y 6 ; pendiente de la recta tangente a tiempo cero de los polinomios y comparación con el coeficiente que acompaña la potencia de orden 1 en el polinomio; velocidad inicial media a diferentes intervalos de tiempo, encontrándose que ante una variación sistemática de la cantidad inicial de peróxido o de carbón, únicamente la velocidad inicial evaluada como velocidad media a los 20 s de reacción se ajusta a una curva suave, siendo este el criterio escogido para establecer el método más adecuado para determinar las velocidades iniciales.

Por consiguiente, para todo el estudio cinético de la DCPH la velocidad inicial siempre se evaluó como la velocidad media inicial a 20 s de reacción. Cada experimento se realizó como mínimo por triplicado, reportando como valor obtenido el promedio aritmético. Sin embargo en algunos experimentos se realizaron hasta cinco repeticiones para garantizar, en todos los casos, que la desviación porcentual con respecto a la media no fuera superior al $5 \%$.

\section{RESULTADOS Y DISCUSIÓN}

\section{Variación de la relación masa volumen de carbón activado}

En la Figura 2 se aprecia como varía la velocidad inicial de reacción en función de la relación masa-volumen de carbón activado (CAT), cuando la concentración inicial de peróxido es, (a) 1,60×10-1 mol L-1 y (b) $7,92 \times 10^{-2} \mathrm{~mol} \mathrm{~L}^{-1}$, a una temperatura de $298 \mathrm{~K}$.

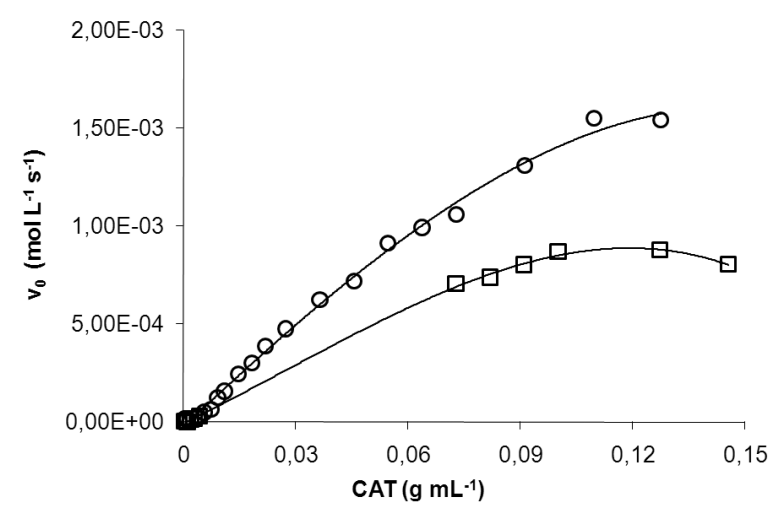

Figura 2. Velocidad inicial de reacción de la DCPH en función de la CAT. (O) $\left[\mathrm{H}_{2} \mathrm{O}_{2}\right]_{0}=1,61 \times 10^{-1} \mathrm{~mol} \mathrm{~L}^{-1},(\square)\left[\mathrm{H}_{2} \mathrm{O}_{2}\right]_{0}=7,92 \times 10^{-2} \mathrm{~mol} \mathrm{~L}^{-1} . \mathrm{T}=298 \mathrm{~K}$

Los datos anteriores se evaluaron mediante el método diferencial, asumiendo inicialmente que la DCPH se puede describir mediante una ley de velocidad como la de la Ecuación 3

$$
v_{\left[\mathrm{H}_{2} \mathrm{O}_{2}\right]_{o}}=k_{v e l} \mathrm{CAT}^{n_{1}}\left[\mathrm{H}_{2} \mathrm{O}_{2}\right]^{n_{2}}
$$

obteniéndose que el orden de reacción con respecto a la relación masa-volumen de carbón activado es uno y que la constante aparente de velocidad, $k_{v e l}$, es aproximadamente $0,11 \mathrm{~mL} \mathrm{~g}^{-1} \mathrm{~s}^{-1}$ a $298 \mathrm{~K}(0,12$ $\mathrm{mL} \mathrm{g}^{-1} \mathrm{~s}^{-1}$ cuando $\left[\mathrm{H}_{2} \mathrm{O}_{2}\right]_{0}=1,60 \times 10^{-1} \mathrm{~mol} \mathrm{~L}^{-1}$ y $0,10 \mathrm{~mL} \mathrm{~g}^{-1} \mathrm{~s}^{-1}$ cuando $\left.\left[\mathrm{H}_{2} \mathrm{O}_{2}\right]_{0}=7,92 \times 10^{-2} \mathrm{~mol} \mathrm{~L}^{-1}\right)$.

\section{Variación de la concentración inicial de peroxido}

En la Figura 3 se aprecia la variación de la velocidad inicial de reacción en función de la concentración inicial de peróxido de hidrógeno, cuando la relación masa-volumen de carbón activado (CAT) es: (a) $1,0 \times 10^{-3} \mathrm{~g} \mathrm{~mL}^{-1}$, (b) $2,0 \times 10^{-3} \mathrm{~g} \mathrm{~mL}^{-1} \mathrm{y}$ (c) $4,0 \times 10^{-3} \mathrm{~g} \mathrm{~mL}^{-1}$, a una temperatura de $298 \mathrm{~K}$.

Los datos de la figura anterior se evaluaron mediante el método diferencial, según la Ecuación 3, obteniendo que el orden de reacción con respecto a la concentración inicial de peróxido de hidrógeno es aproximadamente 0,9 y que la constante aparente de velocidad es aproximadamente $0,11 \pm 0,01 \mathrm{~mL} \mathrm{~g}^{-1} \mathrm{~s}^{-1}$ a $298 \mathrm{~K},\left(0,11 \mathrm{~mL} \mathrm{~g}^{-1}\right.$ para CAT $=1,00 \times 10^{-3} \mathrm{~g} \mathrm{~mL}^{-1} ; 0,10 \mathrm{~mL} \mathrm{~g}^{-1}$ para CAT $=2,00 \times 10^{-3} \mathrm{~g} \mathrm{~mL}^{-1}$; $0,12 \mathrm{~mL} \mathrm{~g}^{-1}$ para CAT $=4,00 \times 10^{-3} \mathrm{~g} \mathrm{~mL}^{-1}$ ).

\section{Efecto de la temperatura}

En la Figura 4 se muestra el efecto de la temperatura en las velocidades iniciales de reacción, en función de la concentración 


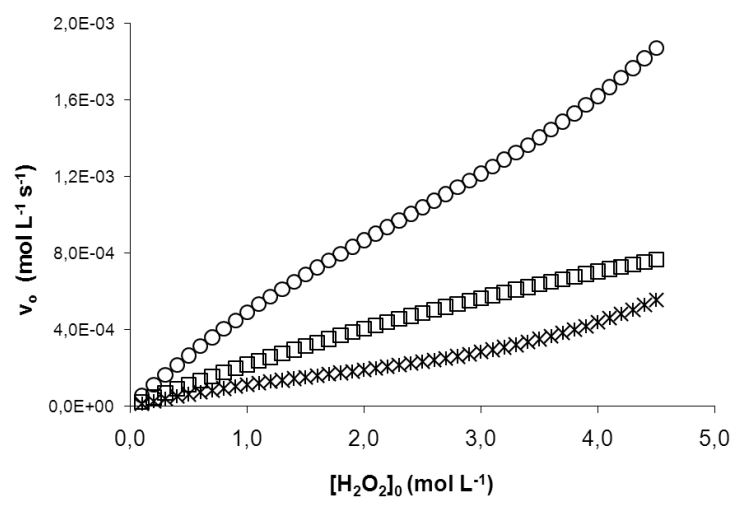

Figura 3. Velocidades iniciales de reacción de la DCPH en función de la concentración inicial de peróxido para diferentes CAT. (*) CAT $=1,00 \times 10^{-3} \mathrm{~g} \mathrm{~mL}^{-1}$, (口) $C A T=2,00 \times 110^{-3} \mathrm{~g} \mathrm{~mL}^{-1}$, (O) $C A T=4,00 \times 10^{-3} \mathrm{~g} \mathrm{~mL}^{-1} . T=298 \mathrm{~K}$

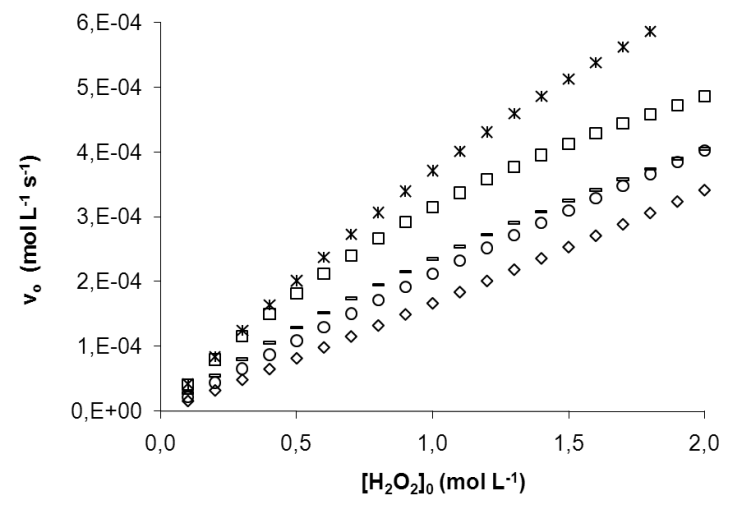

Figura 4. Efecto de la temperatura en la velocidad inicial de la DCPH en función de la $\left[\mathrm{H}_{2} \mathrm{O}_{2}\right]_{0^{*}}(\diamond) T=293 \mathrm{~K}$, (О) $T=298 \mathrm{~K},(-) \mathrm{T}=303 \mathrm{~K}$, (口) $\mathrm{T}$ $=308 \mathrm{~K},(*) \mathrm{T}=313 \mathrm{~K} . \mathrm{CAT}=2,00 \times 10^{-3} \mathrm{~g} \mathrm{~mL}^{-1}$

inicial de peróxido de hidrógeno cuando la relación masa-volumen de carbón activado es $2,00 \times 10^{-3} \mathrm{~g} \mathrm{~mL}^{-1}$.

Asumiendo que la ley de velocidad de la Ecuación 3 es válida, los datos cinéticos de la Figura 4 se evaluaron mediante el método diferencial con el propósito de calcular la constante cinética en función de la temperatura (Tabla 1).

En la Tabla 1 se muestra que el orden de reacción se aleja de uno a medida que la temperatura aumenta de 293 a $313 \mathrm{~K}$, dando la primera evidencia de la complejidad en el mecanismo de reacción, como se presentará mas adelante.

A partir de los datos de la Tabla 1 se estimó que la energía de activación asociada a la DCPH, según la ecuación de Arrhenius, es de aproximadamente $27,6 \mathrm{~kJ} \mathrm{~mol}^{-1}$ en el intervalo de concentración estudiado.

\section{Modelos cinéticos alternativos}

La Figura 1 muestra claramente que durante la descomposición del peróxido de hidrógeno la superficie del carbón activado se modifica químicamente, disminuyendo los sitios activos donde puede ocurrir la descomposición.

Algunos autores consideran que el carbón activado actúa únicamente como un catalizador de transferencia electrónica para la DCPH, según el siguiente esquema de reacción: ${ }^{1-3,9-10}$

$\mathrm{CA}^{+}+\mathrm{I} \rightarrow \mathrm{CA}+2 \mathrm{H}_{2} \mathrm{O}+\mathrm{O}_{2}$

$\mathrm{CA}+2 \mathrm{H}_{2} \mathrm{O}_{2} \rightarrow \mathrm{CA}^{+}+\mathrm{I}$
Tabla 1. Variación de la constante cinética de reacción en función de la temperatura y verificación del orden de reacción con respecto al peróxido de hidrógeno. Se muestran las ecuaciones que linealizan los datos experimentales de la Figura 4 y el respectivo coeficiente de correlación, $\mathrm{r}^{2}$

\begin{tabular}{|c|c|c|c|c|}
\hline $\mathrm{T}(\mathrm{K})$ & Ecuación lineal & $r^{2}$ & $\begin{array}{l}\text { Orden de } \\
\text { reacción }\end{array}$ & $\begin{array}{c}k_{v e l} \\
\left(\mathrm{~mL} \mathrm{~s}^{-1} \mathrm{~g}^{-1}\right)\end{array}$ \\
\hline 293 & $\begin{array}{c}\operatorname{In}\left(\mathrm{V}_{0}\right)= \\
1,02 \operatorname{In}\left(\left[\mathrm{H}_{2} \mathrm{O}_{2}\right]_{0}\right)-8,69\end{array}$ & 0,999 & 1,0 & 0,084 \\
\hline 298 & $\begin{array}{c}\operatorname{In}\left(\mathrm{V}_{0}\right)= \\
0,86 \operatorname{In}\left(\left[\mathrm{H}_{2} \mathrm{O}_{2}\right]_{0}\right)-8,48\end{array}$ & 0,999 & 0,9 & 0,10 \\
\hline 303 & $\begin{array}{c}\operatorname{In}\left(\mathrm{V}_{0}\right)= \\
1,02 \operatorname{In}\left(\left[\mathrm{H}_{2} \mathrm{O}_{2}\right]_{0}\right)-8,40\end{array}$ & 0,998 & 0,9 & 0,11 \\
\hline 308 & $\begin{array}{c}\operatorname{In}\left(\mathrm{V}_{0}\right)= \\
0,76 \mathrm{In}\left(\left[\mathrm{H}_{2} \mathrm{O}_{2}\right]_{0}\right)-8,14\end{array}$ & 0,988 & 0,8 & 0,15 \\
\hline 313 & $\begin{array}{c}\operatorname{In}\left(\mathrm{V}_{0}\right)= \\
0,85 \operatorname{In}\left(\left[\mathrm{H}_{2} \mathrm{O}_{2}\right]_{0}\right)-7,96\end{array}$ & 0,995 & 0,8 & 0,18 \\
\hline
\end{tabular}

Un esquema de reacción como el anterior justifica la Ecuación 3 , de donde finalmente se llega a la ley de pseudo-primer orden para la descomposición del peróxido de hidrógeno.

A diferencia de lo anterior, proponemos un posible esquema de reacción, Esquema 1, que tenga en cuenta la modificación química del carbón, para a partir de ella proponer posibles leyes de velocidad que describan de una manera más precisa la tendencia experimental de los datos cinéticos obtenidos en este trabajo para la DCPH.

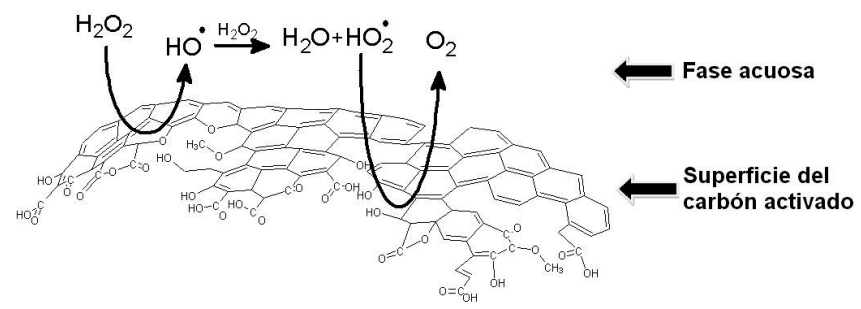

Esquema 1. Esquema de reacción para la DCPH. El $\mathrm{H}_{2} \mathrm{O}_{2}$ reacciona, sobre la superficie de carbón activado con grupos básicos o reductores iniciando una reacción en cadena mediada por radicales libres

Las consideraciones realizadas para llegar a este esquema de reacción son las siguientes: la molécula de peróxido de hidrógeno se adsorbe sobre la superficie de carbón activado; el peróxido de hidrógeno adsorbido reacciona en sitios activos donde haya grupos funcionales formando sobre la superficie intermediarios de reacción; los intermediarios se desorben y difunden hacia la fase fluida, donde por reacción con otras moléculas de peróxido de hidrógeno puede formar nuevos intermediarios de reacción; los nuevos intermediarios se difunden hacia la superficie del carbón, reaccionando con ésta, para producir finalmente oxígeno molecular dejando químicamente modificada la superficie del carbón.

Las reacciones R1 a R5 corresponden a las consideraciones anteriormente realizadas:

$$
\begin{aligned}
& \mathrm{H}_{2} \mathrm{O}_{2}{ }^{(\mathrm{ac})}+\mathrm{S} \stackrel{\mathrm{K}_{\mathrm{eq}}}{=}\left(\mathrm{H}_{2} \mathrm{O}_{2}-\mathrm{S}\right)^{(\mathrm{ad})} \\
& \mathrm{S}+\left(\mathrm{H}_{2} \mathrm{O}_{2}-\mathrm{S}\right)^{(\mathrm{add})} \stackrel{\mathrm{k}_{1}}{\longrightarrow} \mathrm{HO}_{\text {(ac) }}^{\cdot}+\mathrm{S}^{\circ}+\mathrm{S}-\mathrm{OH} \\
& \mathrm{H}_{2} \mathrm{O}_{2(\text { (ac) }}+\mathrm{HO}_{(\mathrm{ac})}^{\circ} \stackrel{\mathrm{K}_{2}}{\rightleftharpoons} \mathrm{HO}_{2^{(\mathrm{ac})}}^{\circ}+\mathrm{H}_{2} \mathrm{O}_{(\mathrm{l})} \\
& \mathrm{S}^{\circ}+\mathrm{HO}_{2}^{\cdot(\mathrm{ac})} \stackrel{\mathrm{k}_{3}}{\longrightarrow} \mathrm{O}_{2^{(\mathrm{g})}}+\mathrm{S}-\mathrm{H}
\end{aligned}
$$




$$
\mathrm{S}^{\cdot}+\mathrm{HO}_{\text {(ac) }}^{\cdot} \stackrel{\mathrm{k}_{4}}{=} \mathrm{S}-\mathrm{OH}
$$

donde $\mathrm{S}$ representa el sitio activo de la superficie del carbón, $\mathrm{HO}^{\circ} \mathrm{y}$ $\mathrm{HO}_{2} \cdot$ los intermediarios de reacción.

Otra opción para analizar, es considerar la modificación química de la superficie del carbón por adsorción disociativa del peróxido de hidrógeno como lo muestran las reacciones R6 y R7,

$\mathrm{H}_{2} \mathrm{O}_{2 \text { (ac) }}+2 \mathrm{~S} \stackrel{\mathrm{keq}^{*}}{=} 2 \mathrm{~S}-\mathrm{OH}$

$\mathrm{H}_{2} \mathrm{O}_{2(\text { (ac) }}+\mathrm{S}-\mathrm{OH} \stackrel{\mathrm{k}^{*} \mathrm{D}}{\rightleftharpoons} \mathrm{O}_{2}{ }^{(\mathrm{g})}+\mathrm{S}-\mathrm{H}^{+} \mathrm{H}_{2} \mathrm{O}_{(1)}$

Para el tratamiento cinético de los datos, teniendo en cuenta las reacciones $\mathrm{R} 1$ a $\mathrm{R} 7$, se proponen las siguientes leyes de velocidad, según si el paso determinante es: la adsorción del peróxido de hidrógeno sobre la superficie del carbón (R1). Entonces la ecuación (3) se modifica para expresar la concentración de peróxido con una ecuación tipo Langmuir que considera la fracción adsorbida de peróxido, Ecuación 4:

$v_{\left[H_{2} O_{2}\right]_{0}}=\frac{k_{e q}\left[H_{2} O_{2}\right]_{0}}{1+k_{e q}\left[H_{2} \mathrm{O}_{2}\right]_{0}} C A T$

la reacción entre especies adsorbidas. Entonces la Ecuación 3 se modifica según la Ecuación de Langmuir-Hinshelwood (2), la reacción entre especies en solución y especies adsorbidas. Entonces la Ecuación 3 se modifica según la Ecuación de Rideal-Eley, Ecuación 5:

$v_{\left[\mathrm{H}_{2} \mathrm{O}_{2}\right]_{0}}=\frac{k_{v e l}\left[\mathrm{H}_{2} \mathrm{O}_{2}\right]_{0}^{2}}{1+k_{e q}\left[\mathrm{H}_{2} \mathrm{O}_{2}\right]_{0}} C A T$

la adsorción disociativa del peróxido de hidrógeno sobre la superficie del carbón. Entonces la Ecuación 3 se modifica según la Ecuación de Langmuir-Hinshelwood disociativa, Ecuación 6:

$v_{\left[H_{2} \mathrm{O}_{2}\right]_{0}}=\frac{k_{v e l} k^{1 / 2}{ }_{e q}\left[\mathrm{H}_{2} \mathrm{O}_{2}\right]_{0}^{1 / 2}}{1+k^{1 / 2}{ }_{e q}\left[\mathrm{H}_{2} \mathrm{O}_{2}\right]_{0}^{1 / 2}} C A T$

Las Ecuaciones 2, 4, 5 y 6 se evaluaron para los datos experimentales de velocidades iniciales de reacción en función de las concentraciones iniciales de reactivos, encontrando que las ecuaciones tipo Langmuir, Ecuaciones 2 y 4, describen la tendencia experimental de los datos, sin embargo únicamente la ecuación de Langmuir-Hinshelwood es la que ajusta con mayor precisión los datos experimentales, cuando se hacen variaciones en la concentraciones iniciales de peróxido y de carbón. Los resultados se muestran en la Figura 5 y Tabla 2.

Después del resultado anterior, los datos de la Figura 4, donde se muestra el efecto de la temperatura en las velocidades iniciales de la DCPH, se ajustaron con la Ecuación de Langmuir-Hinshelwood para determinar las constantes cinéticas (Figura 6 y Tabla 3 ).

A partir de los datos de $k_{v e l}$ en la Tabla 3, se calcula que la energía de activación es de aproximadamente $40,4 \mathrm{~kJ} \mathrm{~mol}^{-1}$ según el modelo de Arrhenius.

En la Tabla 2 se muestra que la constante $k_{v e l}$ da un valor más o menos consistente entre si ante las variaciones de la relación masavolumen de carbón activado, y en un orden de magnitud comparable a la constante de velocidad previamente calculada para la Ecuación 3. Sin embargo la magnitud de la constante $k_{h}$ varía de manera significativa en esos experimentos, lo cual indica que si bien el modelo de Langmuir-Hinshelwood describe la tendencia de los datos experimentales, no es preciso en la descripción del mecanismo de reacción.

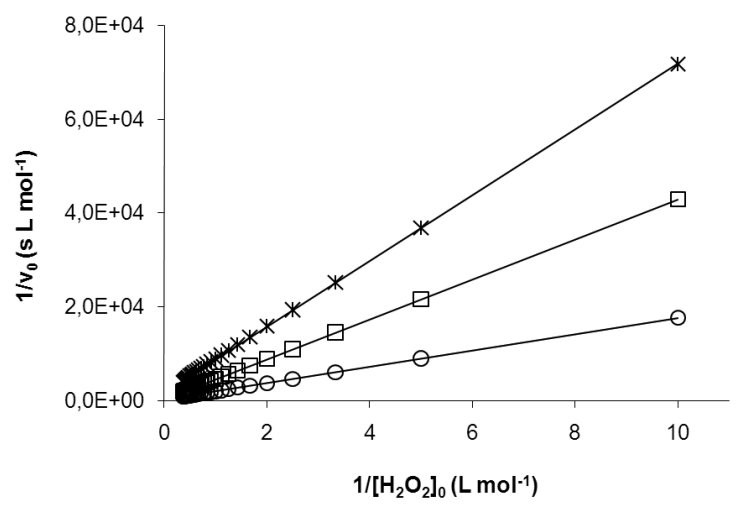

Figura 5. Linealización del modelo de Langmuir-Hinshelwood(Ecuación 2), para los datos experimentales de velocidad inicial de reacción en función de la $\left[\mathrm{H}_{2} \mathrm{O}_{2}\right]_{0}$ a diferentes CAT. (*) $C A T=1,00 \times 10^{-3} \mathrm{~g} \mathrm{~mL}^{-1}$, ( $\square$ ) CAT $=2,00 \times 10^{-3}$ $\mathrm{g} \mathrm{mL}$, (О) $C A T=4,00 \times 10^{-3} \mathrm{~g} \mathrm{~mL}^{-1} . T=298 \mathrm{~K}$

Tabla 2. Constantes de velocidad $k_{v e l}$ y de equilibrio $k_{h}$ para la descomposición catalizada del peróxido de hidrógeno según el modelo de Langmuir-Hinshelwood, a 298 K. En paréntesis el símbolo que identifica los datos experimentales de la Figura 5

\begin{tabular}{lcc}
\hline$C A T\left(\mathrm{~g} \mathrm{~mL}^{-1}\right)$ & $k_{v e l}\left(\mathrm{~mL} \mathrm{~s}^{-1} \mathrm{~L}^{-1}\right)$ & $k_{h}\left(\mathrm{~L} \mathrm{~mol}^{-1}\right)$ \\
\hline $1,000 \times 10^{-3}(*)$ & 0,25 & 0,14 \\
$2,000 \times 10^{-3}(+)$ & 0,053 & 0,11 \\
$4,000 \times 10^{-3}(\mathrm{O})$ & 0,16 & 0,14 \\
\hline
\end{tabular}

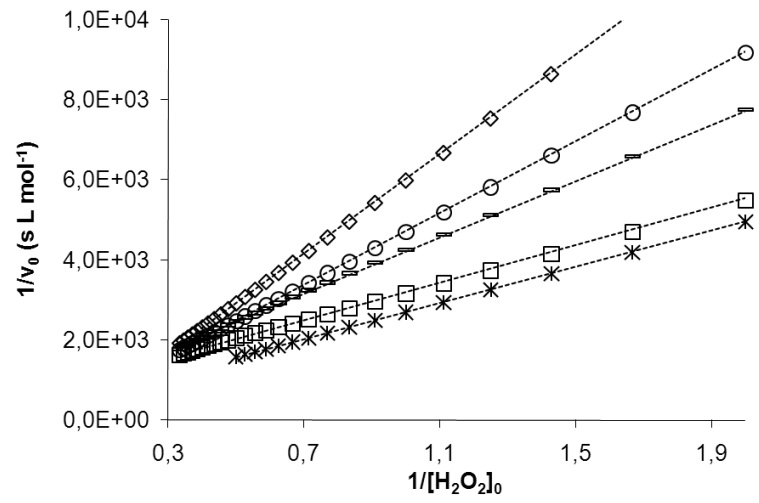

Figura 6. Linealización del modelo de Langmuir-Hinshelwood (Ecuación 2) en función de la temperatura, para los datos de velocidad inicial de la DCPH en función de la concentración inicial de peróxido de hidrógeno. ( $\diamond) T=$ $293 \mathrm{~K}$, (О) $T=298 \mathrm{~K}$, (-) $T=303 \mathrm{~K}$, ( $\square$ ) $T=308 \mathrm{~K}$, (*) $T=313 \mathrm{~K}$. CAT $=2,000 \times 10^{-3} \mathrm{~g} \mathrm{~mL}^{-1}$

Los datos de la Tabla 3 muestran que la constante $k_{v e l}$ varía más significativamente con la temperatura al compararse con los datos de la Tabla 1, lo cual es consecuencia de las diferencias en los modelos cinéticos utilizados. Es por esto que la energía de activación obtenida da un valor mucho mayor que el obtenido a partir de la Ecuación 3.

Una energía de activación de $27,6 \mathrm{~kJ} \mathrm{~mol}^{-1}$ es consistente con valores reportados para la DCPH en fase homogénea utilizando una ley cinética de pseudo-primer orden $\left(11 \mathrm{a} 26 \mathrm{~kJ} \mathrm{~mol}^{-1}\right){ }^{11,12} \mathrm{Sin}$ embargo el sistema que se estudió es de naturaleza heterogénea y para este tipo de sistemas se encuentra reportado en literatura un solo trabajo en el cual se estudio el efecto de la temperatura en la DCPH sobre óxidos de magnesio, ${ }^{5,6}$ obteniendo que la energía de activación se encuentra en el intervalo de 12 a $77 \mathrm{~kJ} \mathrm{~mol}^{-1}$, valores obtenidos a 
Tabla 3. Constantes del modelo de Langmuir-Hinshelwood en función de la temperatura, obtenidas a partir de los datos de la Figura 6. Se muestran las ecuaciones que linealizan los datos experimentales y el respectivo coeficiente de correlación, $\mathrm{r}^{2}$

\begin{tabular}{|c|c|c|c|c|}
\hline $\mathrm{T}(\mathrm{K})$ & Ecuación lineal & $r^{2}$ & $\begin{array}{c}k_{v e l} \\
\left(\mathrm{~mL} \mathrm{~s}^{-1} \mathrm{~g}^{-1}\right)\end{array}$ & $\begin{array}{c}k_{n} \\
\left(\mathrm{~L} \mathrm{~mol}^{-1}\right)\end{array}$ \\
\hline 293 & $\begin{array}{c}\mathrm{V}_{0}^{-1}= \\
6207 \times\left[\mathrm{H}_{2}{ }_{2}\right]_{0}^{-1}-152,7\end{array}$ & 1,000 & 0,080 & 0,00 \\
\hline 298 & $\begin{array}{c}\mathrm{V}_{0}^{-1}= \\
4481 \times\left[\mathrm{H}_{2} \mathrm{O}_{2}\right]_{0}^{-1}+258,7\end{array}$ & 1,000 & 0,11 & 0,053 \\
\hline 303 & $\begin{array}{c}\mathrm{V}_{0}^{-1}= \\
3534 \times\left[\mathrm{H}_{2} \mathrm{O}_{2}\right]_{0}^{-1}+603,4\end{array}$ & 0,999 & 0,14 & 0,20 \\
\hline 308 & $\begin{array}{c}\mathrm{V}_{0}^{-1}= \\
2365 \times\left[\mathrm{H}_{2} \mathrm{O}_{2}\right]_{0}^{-1}+788,2\end{array}$ & 1,000 & 0,21 & 0,36 \\
\hline 313 & $\begin{array}{c}\mathrm{V}_{0}^{-1}= \\
2264 \times\left[\mathrm{H}_{2} \mathrm{O}_{2}\right]_{0}^{-1}+458,5\end{array}$ & 1,000 & 0,22 & 0,20 \\
\hline
\end{tabular}

partir de una ley de pseudo-primer orden y con datos experimentales bastante irreproducibles.

Por otra parte el valor de $40 \mathrm{~kJ} \mathrm{~mol}^{-1}$ que hemos obtenido mediante el modelo de Langmuir-Hinshelwood parece ser un valor más acorde a las reacciones químicas en fase heterogénea. ${ }^{13}$

\section{CONCLUSIONES}

Experimentalmente se da evidencia de que la superficie del carbón activado se modifica químicamente durante la descomposición del peróxido de hidrógeno, igualmente se presenta evidencia de que los sitios activos corresponden a grupos funcionales básicos o reductores, ya que cuando el carbón activado fue tratado con oxidantes fuertes como el permanganato de potasio y el peróxido de hidrógeno, la velocidad de descomposición del peróxido de hidrógeno disminuyó de manera significativa (Figura 1). El amplio conjunto de datos experimentales obtenidos a partir del estudio de la velocidad inicial de la DCPH, en un intervalo amplio de concentraciones iniciales de peróxido y cantidad inicial de carbón activado, al igual que el efecto de la temperatura, se estudió mediante una ley de velocidad típica de una reacción en fase homogénea, Ecuación 3, obteniendo que el orden de reacción para cada uno de los reactivos es 1,0, con una energía de activación de aproximadamente $27 \mathrm{~kJ} \mathrm{~mol}^{-1}$. Si bien estos resultados justifican una ley de pseudo-primer orden para la DCPH, desconocen la naturaleza heterogénea del sistema estudiado. Finalmente se ha mostrado que los datos experimentales se pueden tratar, satisfactoriamente, con una ecuación cinética que tenga en cuenta la naturaleza del sistema, como es la Ecuación de Langmuir-Hinshelwood. En estas condiciones la energía de activación es aproximadamente 40 $\mathrm{kJ} \mathrm{mol}^{-1}$. Este trabajo muestra el procedimiento a seguir cuando la descomposición del peróxido de hidrógeno se utiliza como técnica para caracterizar la actividad catalítica de sólidos modificados.

\section{AGRADECIMIENTOS}

A la Dirección Nacional de Investigaciones de la Universidad Nacional de Colombia por la financiación a través del proyecto 20601002443. E. Paternina y J. M. Arias agradecen a la Universidad Nacional de Colombia por el apoyo económico brindado a través del cargo auxiliar docente.

\section{REFERENCIAS}

1. Puri, B. R.; Kalra, K. C.; Carbon 1971, 9, 313.

2. Copper, C. L.; Koubek E.; J. Chem. Educ. 1998, 75, 87.

3. Oliveira, L.; Silva, C.; Yoshida, M.; Lago, R.; Carbon 2004, 42. 2279.

4. Salem, I. A.; El-Maazawi, M.; Zaki, A. B.; Int. J. Chem. Kinet. 2000, 32, 643.

5. Hiroki, A.; LaVerne, J. A.; J. Phys. Chem. B 2005, 109, 3364.

6. Hasan, M. A.; Zaki, M. I.; Pasupulety, L.; Kumari, K.; Appl. Catal. A: General 1999, 181, 171.

7. Lin, S. S.; Gurol, M. D.; Environm. Sci. Technol. 1998, 32, 1417.

8. Huang, H.; Lu, M.; Chen, J.; Lee, C.; Chemosphere 2003, 51, 935.

9. Huang, H; Lu, M.; Chen, J.; Chemosphere 2001, 35, 2291.

10. Georgi, A.; Kopinke, D.; Appl. Catal. B: Environm. 2005, 58, 9.

11. Copper, C. L.; Koubek, E.; Thermochim. Acta 2005, 437, 48.

12. Kavitha, V.; Palanivelu, K.; Water Res. 2005, 39, 3062.

13. Rouqueroul, F.; Rouqueroul, J.; Sing, K.; Adsorption by powders and porous solid: principles, methodology and applications, Academic Press: London, 1999, p. 467. 